\title{
Small cell carcinoma of the rectum: A report of imaging results from four cases
}

\author{
JIAN ZHOU, JINGXIAN SHEN, HUALI MA, YUN ZHANG，MEILI SUN，LIE ZHENG and YANCHUN LV \\ Medical Imaging and Minimally Invasive Interventional Center, \\ State Key Laboratory of Oncology in South China, Collaborative Innovation Center for Cancer Medicine, \\ Sun Yat-sen University Cancer Center, Guangzhou, Guangdong 510060, P.R. China
}

Received February 24, 2015; Accepted December 3, 2015

DOI: $10.3892 / \mathrm{ol} .2016 .4136$

\begin{abstract}
Small cell carcinoma of the rectum is extremely rare. The present study aimed to improve the recognition of computed tomography (CT) and magnetic resonance imaging (MRI) features observed in patients with small cell carcinoma of the rectum. A total of 4 cases of patients with histologically confirmed small cell carcinoma of the rectum were retrospectively reviewed. The imaging features assessed consisted of the thickening of the rectal wall, local tumor invasion, CT density, MRI signal intensity, lesion texture, contrast enhancement characteristics, lymphadenopathy and distant metastasis. All 4 patients displayed ring-like rectal wall thickening and hypodensity in comparison to the gluteus muscles on non-enhanced CT images. MRI demonstrated isointensity on T1-weighted images and hyperintensity on T2-weighted images. Thickening of the left wall of the rectum with patchy low attenuation and the presence of an exophytic mass was observed in 1 patient. Local tumor invasion was observed in the perirectal fat spaces of 4 patients, in the prostate gland and seminal vesicle of 1 patient, and in the left seminal vesicle of 1 patient. Heterogeneous attenuation was exhibited on non-enhanced CT images in 3 lesions, and 1 lesion exhibited heterogeneous intensity on non-enhanced MRI images. All the lesions observed demonstrated strong enhancement following contrast imaging. Lymphadenopathy was observed in 4 patients, liver metastasis in 3 patients, pulmonary metastasis in 2 patients and multiple bone metastases in 1 patient. Overall, small cell carcinoma of the rectum appeared to be more likely to metastasize to the liver, pulmonary, lymph
\end{abstract}

Correspondence to: Dr Jingxian Shen, Medical Imaging and Minimally Invasive Interventional Center, State Key Laboratory of Oncology in South China, Collaborative Innovation Center for Cancer Medicine, Sun Yat-sen University Cancer Center, 651 Dongfeng East Road, Guangzhou, Guangdong 510060, P.R. China

E-mail: shenjx@sysucc.org.cn

Key words: small cell carcinoma, rectum, computed tomography, magnetic resonance imaging nodes and bone. Distinguishing features of small cell carcinoma of the rectum were noted as a thickening rectal wall, the presence of soft-tissue tumors, local tumor invasion, lymphadenopathy and distant metastases. Pre-operative CT and MRI are required as an aid in selecting the correct treatment plan and for the prognosis assessment of patients.

\section{Introduction}

Small cell carcinoma is a frequently occurring pulmonary neoplasm that may also be located in extrapulmonary sites, including the salivary glands, pharynx, larynx, thymus, esophagus, stomach, small bowel, colon, rectum, gallbladder, kidney, uterus, skin and breasts (1). Extrapulmonary small cell carcinoma is histologically identical to pulmonary small cell carcinoma, with rapid local progression and exhibiting early regional and distant metastasis (1). Patients with extrapulmonary small cell carcinoma frequently have a poor prognosis, even if diagnosed at an early stage (2). Small cell carcinoma of the large bowel is extremely rare, representing $\sim 0.2 \%$ of all large bowel malignancies (3). To date, there have been $\sim 40$ cases of extrapulmonary small cell carcinoma of the rectum reported in the literature (4), the majority of which are focused on the clinical and pathological features, treatment and prognosis of the disease.

The pathological features of extrapulmonary small cell carcinoma of the rectum are similar to small-cell neuroendocrine carcinomas in other sites, exhibiting closely packed cells with scanty cytoplasm, nuclear pleomorphism, a high mitotic rate and necrosis (4). Neuroendocrine differentiation can be demonstrated by immunohistochemical methods or electron microscopy (2). The differential diagnosis includes metastatic lung small cell carcinoma, which can only be excluded clinically; other small cell malignancies that may occur in this region, such as the more common basaloid or cloacogenic carcinoma, lymphoma, embryonal rhabdomyosarcoma and amelanotic melanoma; and other neuroendocrine tumors, such as carcinoid (4). Long survival (10-20 years) has been reported following resection of the primary tumor, although a median survival of 5-11 months is most commonly reported (4). Computed tomography (CT) and magnetic resonance imaging (MRI), as well as colorectal colonoscopy may be used in the diagnosis of small cell carcinoma of the rectum $(4,5)$. 
Small cell carcinoma of the rectum is extremely rare. The present study aimed to improve the recognition of CT and MRI features observed in patients with small cell carcinoma of the rectum by reporting the radiological features of 4 patients with surgically and pathologically diagnosed small cell carcinoma of the rectum. In particular, the present study reviews the CT and MRI features used to diagnose the patients.

\section{Patients and methods}

Patients. The cases of 4 patients with pathologically confirmed extrapulmonary small cell carcinoma of the rectum were retrospectively reviewed by the present study. The patients were treated at Sun Yat-sen University Cancer Center (Guangzhou, Guangdong, China) between January 2001 and August 2013. The histological diagnosis of rectal small cell carcinoma was confirmed by endoscopic biopsy or surgery (Miles' operation, exploratory laparotomy and sigmoidostomy). The patients consisted of 3 men and 1 woman (age range, 40-65 years; median age, 51.5 years). The clinical records of the patients were obtained, and patient characteristics, including age, gender, tumor stage, clinical symptoms, digital rectal examination and colonoscopy results, laboratory examinations [carcinoembryonic antigen, cancer antigen 19-9 and neuron-specific enolase (NSE)], treatment and follow-up information were reviewed. Sun Yat-sen University Cancer Center Institutional Review Board approval and written informed patient consent were obtained prior to the commencement of the present study.

Imaging protocol. CT was performed for 3 patients using a Brilliance TM16 abdomen scanner (Philips Medical Systems B.V., Eindhoven, The Netherlands) with the following parameters: 5-mm slice thickness; $120-\mathrm{kV}$ voltage; 200-mA current; and a 256x256 matrix. Following non-enhanced CT imaging, an intravenous bolus of $1.5-2.0 \mathrm{ml} / \mathrm{kg}$ of a non-ionic iodine contrast agent (iopromide; Ultravist ${ }^{\circledR}$; Bayer Plc., Newbury, $\mathrm{UK}$ ) was administered at a rate of 2.5-3.0 $\mathrm{ml} / \mathrm{sec}$. Enhanced CT images were obtained at $60 \mathrm{sec}$ post-injection of the contrast agent.

MRI was performed on 1 patient using a 1.5-Tesla unit (GE Signa CVi system; GE Healthcare Life Sciences, Chalfont, UK) with an abdomen combined coil. The following 7 sequences were obtained: Non-enhanced T1-weighted images (T1WI) in axial plane; non-enhanced T2-weighted images (T2WI) in axial plane; non-enhanced fat-suppressed T2WI in axial and sagittal planes; and contrast-enhanced fat-suppressed T1WI in axial, coronal and sagittal planes. A $0.2-\mathrm{mmol} / \mathrm{kg}$ body weight bolus injection of gadopentetate dimeglumine (Magnevist ${ }^{\circledR}$; Bayer Plc.) was administered to obtain contrast-enhanced sequences.

Imaging analysis. Two experienced radiologists independently evaluated the CT and MRI images. Any disagreements were resolved by consensus. The CT and MRI features evaluated consisted of rectal wall thickening, the presence of a soft-tissue tumor, local tumor invasion, CT density, MRI signal intensity (hypointensity, isointensity or hyperintensity in association with gluteal muscle signals), lesion texture (homogeneous, heterogeneous or necrotic), contrast enhancement characteristics (strong, moderate or poor), lymphadenopathy and distant metastasis. The thick rectal wall was measured in the axial plane and the maximum value was selected. Strong enhancement was selected if the degree of enhancement was stronger than that of the gluteal muscles, moderate enhancement was selected if the degree of enhancement was similar to that of the gluteal muscles and poor enhancement was selected if the degree of enhancement exhibited no clear signal increase. Lymphadenopathy was considered abnormal when the maximal short dimension was $>0.5 \mathrm{~cm}$, or when stronger enhancement was observed compared with that of the adjacent gluteal muscles.

Histology. All 4 patients underwent electronic enteroscopic biopsy. The specimens were cut to a width of $3.5 \mu \mathrm{m}$, and examined by microscopic and immunohistochemical analyses in the Pathology Department of Sun Yat-sen University Cancer Center. Immunohistochemical examinations included synaptophysin, cluster of differentiation 56 (CD56), chromogranin A and NSE. The specimens were examined microscopically by hematoxylin and eosin $(\mathrm{H} \& \mathrm{E})$ staining.

\section{Results}

Clinical characteristics. The most common clinical symptoms observed in the patients were tenesmus ( $n=2$; patients 2 and 3 ), increased frequency of defecation ( $n=2$; patients 3 and 4$)$, bloody stools $(n=3$; patients $1-3)$, difficulty in defecation $(n=2$; patients 2 and 4$)$, anal pain ( $n=1$; patient 1$)$ and dysuresia $(n=1$; patient 4$)$. In total, 1 out of the 4 patients was stage IIIC (patient 1), 1 patient was stage IVA (patient 2) and the remaining 2 patients were stage IVB (patients 3 and 4), according to the National Comprehensive Cancer Network guidelines (6). Blood tests revealed that 1 out of 4 patients (patient 1) exhibited an abnormal carcinoembryonic antigen level of $8.19 \mathrm{ng} / \mathrm{ml}$ (normal range, 0-5 ng/ml). The 2 patients who were tested for cancer antigen 19-9 possessed normal levels (patient 1, 8.6 U/ml; patient 2, $6.9 \mathrm{U} / \mathrm{ml}$; normal range, 0-35 U/ml). Blood NSE was evaluated in 1 patient (patient 3), at an abnormal level of $114.8 \mathrm{ng} / \mathrm{ml}$ (normal range, $0.0-15.2 \mathrm{ng} / \mathrm{ml}$ ). In total, 1 patient underwent Miles' operation (patient 1), 1 patient underwent concurrent chemotherapy and radiotherapy (patient 2), 1 patient underwent an exploratory laparotomy, sigmoidostomy and post-operative chemotherapy (patient 3 ), and 1 patient did not receive any treatment (patient 4 ).

Imaging findings. The CT and MRI results are summarized in Table I. All 4 patients exhibited ring-like rectal wall thickening, hypodensity compared with the gluteus muscles on non-enhanced CT images, isointensity on T1WI and hyperintensity on T2WI. Thickening of the left wall of the rectum with patchy low attenuation and the presence of an exophytic mass was observed in 1 patient (patient 4; Fig. 1). Local tumor invasion of the perirectal fat spaces was observed in 4 patients. Tumor invasion of the seminal vesicle was exhibited by 1 patient (patient 1), and tumor invasion of the seminal vesicle and prostate gland was exhibited by 1 patient (patient 4). Heterogeneous attenuation was found on non-enhanced CT images in 3 lesions (patients 1, 2 and 4), and 1 lesion exhibited heterogeneous intensity on non-enhanced MRI (patient 3). All the lesions demonstrated strong enhancement following contrast imaging. Lymphadenopathy was observed in 4 patients, pulmonary 


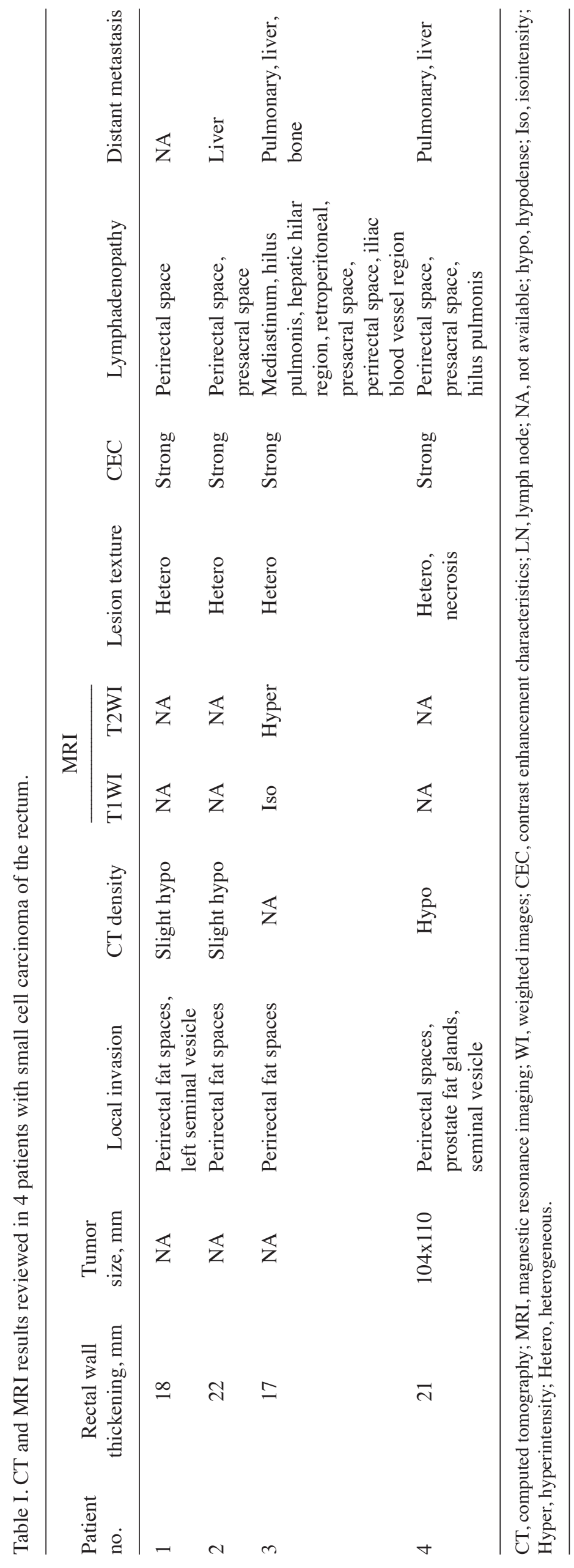



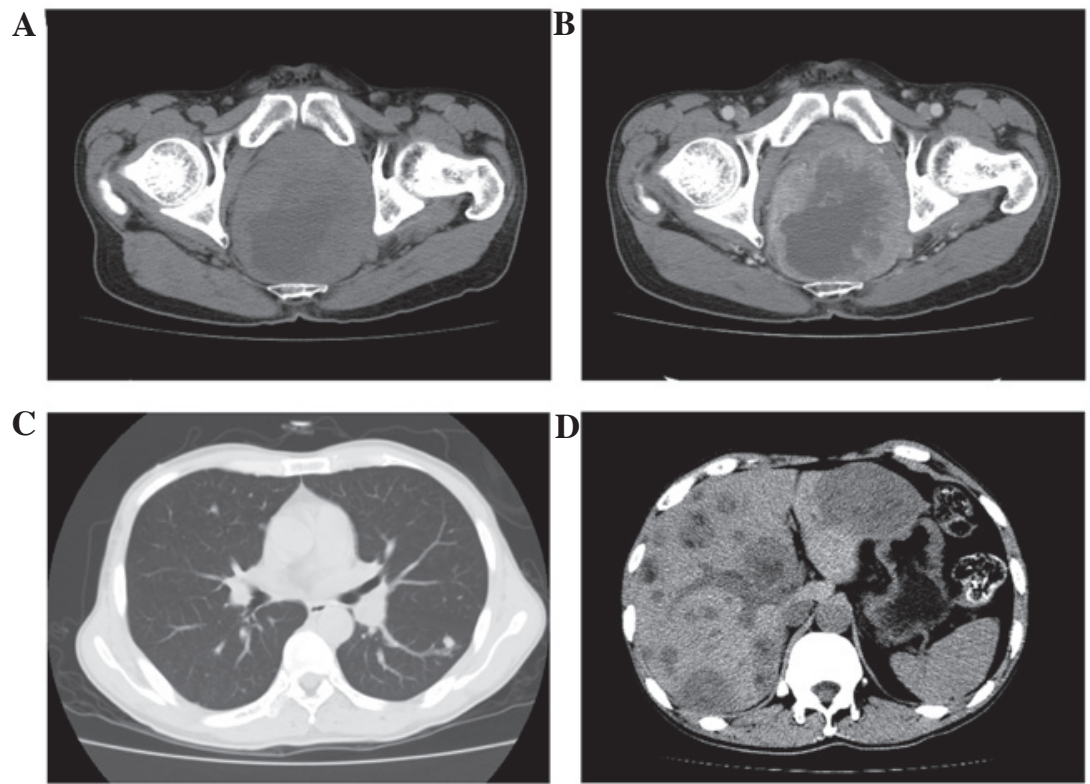

Figure 1. CT scans of a 45-year-old man with small cell carcinoma of the rectum (patient 4). (A) Axial non-enhanced CT scan demonstrating a thickened left wall of the rectum, with patchy low attenuation and the presence of an exophytic mass. Tumor invasion of the prostate glands and seminal vesicle was also observed. (B) Axial contrast-enhanced CT scan demonstrating heterogeneous strong enhancement. (C) Axial CT scan demonstrating the presence of multiple lung metastases. (D) Axial non-enhanced and contrast-enhanced CT scan demonstrating the presence of multiple liver metastases. CT, computed tomography.
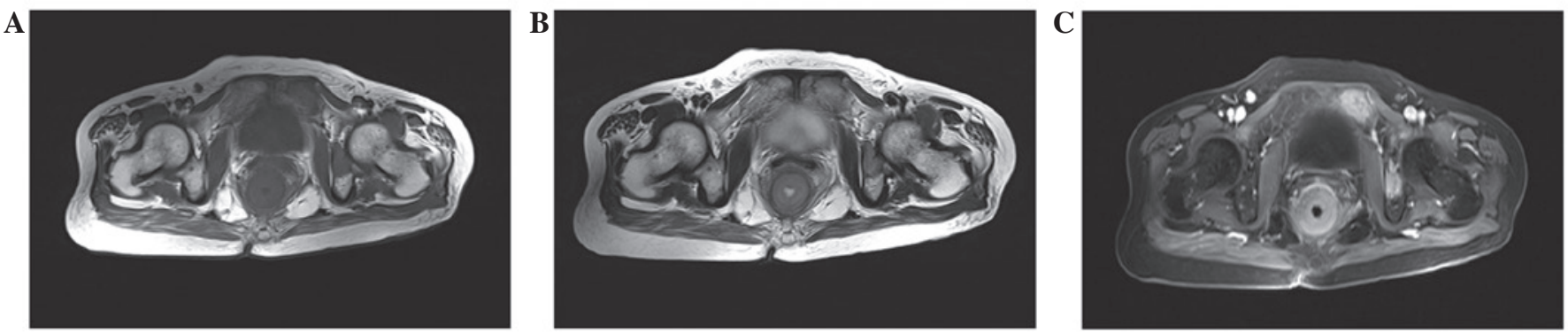

Figure 2. Magnetic resonance images of a 58-year-old woman with small cell carcinoma of the rectum (patient 3). (A) Axial T1WI and (B) axial T2WI demonstrating isointense and hypointense thickening of the rectal wall, respectively, compared with the gluteus muscles. Left pubic bone metastasis was also observed. (C) Axial T1WI fat-suppression demonstrating heterogeneous strong enhancement. WI, weighted images.

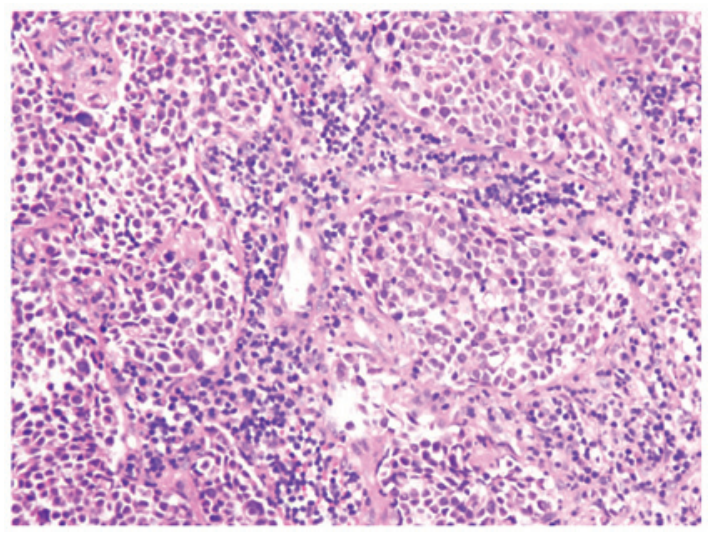

Figure 3. Example of post-operative histopathology revealing small cell carcinoma with lymphatic spread (patient 2). Magnification, x400; stain, hematoxylin and eosin.

metastasis in 2 patients (patients 3 and 4), liver metastasis in 3 patients (patient 2-4) and multiple bone metastases in 1 patient (patient 3 ; Fig. 2).
Pathological findings. All 4 patients were diagnosed with extrapulmonary small cell carcinoma of the rectum using H\&E staining (patient 2; Fig. 3) and immunohistochemistry. Immunohistochemical analysis was performed on 3 post-operative specimens (patients 2-4). Of the total patients, 1 was diagnosed with a mixed cell type, consisting of small cell carcinoma and moderately-differentiated adenocarcinoma (patient 1), observed using H\&E staining. Immunohistochemical examinations revealed that the tumor cells expressed synaptophysin and CD56 in 3 patients (patients 2-4), and chromogranin A and NSE in 2 patients (patients 2 and 3). None of the patients were negative for the antibodies relevant to small carcinoma of the rectum.

\section{Discussion}

The etiology of extrapulmonary small cell carcinoma remains unknown. Certain patients with extrapulmonary small cell carcinoma may have an ectopic production of hormones, including corticotropin, calcitonin, somatostatin and gastrin, 
which is similar to small cell carcinoma of the lung, although clinical manifestations resulting from hormone production are rare (7). The largest series of cases of extrapulmonary small cell carcinoma were retrospectively reviewed from a cancer database in Southeast England over 34 years (8). The study identified 1,600 patients with extrapulmonary small cell carcinoma, of which $33 \%$ were gastrointestinal in origin, followed by genitourinary (20\%), head and neck (11\%) and breast $(10 \%)$ origins. The majority of gastrointestinal small cell carcinoma occurs in the esophagus $(53-71 \%)$ and the colon $(13 \%)(1,9)$. Colorectal small cell carcinoma is an extremely rare tumor, with an incidence of $<0.2 \%$ in all types of colorectal cancers (2). The present study retrospectively reviewed the cases of patients with small cell carcinoma of the rectum, who presented at Sun Yat-sen University Cancer Center between January 2001 and August 2013. A total of 4 patients were identified from the picture archiving and communication system of the center.

In a retrospective study of 64 cases in the USA, the most common locations identified for extrapulmonary small cell carcinoma were the colon and rectum (10). The median age at presentation was 55 years and a slight male predominance was observed. The median survival time ranged between 6 and 12 months for treated patients, down to only weeks for untreated patients. A median survival time of 5 months has also been reported for extrapulmonary small cell carcinoma in an additional study (7). In the present study, the median age at presentation was 51.5 years and the patients consisted of 3 men and 1 woman. In total, 2 patients were lost to follow-up and 2 patients succumbed to the carcinoma $\sim 1$ and 2 months after discharge from hospital, respectively.

Histologically, extrapulmonary small cell carcinoma is identical to pulmonary small cell carcinoma (11). In the current study, all the patients met the histopathological criteria for the diagnosis of extrapulmonary small cell carcinoma, namely the presence of small round or spindle-shaped cells with intensely hyperchromatic nuclei, scant cytoplasm and frequent mitoses $(1,5)$. Immunohistochemically, extrapulmonary small cell carcinoma often expresses synaptophysin and chromogranin A (11). In the present study, the tumors of 3 patients expressed synaptophysin and the tumors of 2 patients expressed chromogranin A and NSE.

Small cell carcinomas are hypothesized to be derived from a pluripotent neuroendocrine stem cell (4). Ihtiyar et al (2) reported a case of tubulovillous adenoma overlaying small cell carcinoma of the rectum. In the present study, 1 tumor was composed of small cell carcinoma and moderately-differentiated adenocarcinoma, suggesting that small cell carcinoma may be derived from stem cells, which has been previously reported in other studies (12-14).

The majority of previous studies concerning CT and MRI results in cases of extrapulmonary small cell carcinoma of the rectum appear to be case reports. Spiliopoulou et al (15) reported a case in which CT confirmed the presence of a large rectal mass extending from the anorectal junction to the mid-rectum, with multiple enlarged perirectal and mesorectal lymph nodes. Ihtiyar et al (2) reported a case in which abdominal tomography revealed the presence of a large rectal neoplasm with multiple lesions, which were possibly metastatic, in the right and left lobes of the liver, and massive enlargement of the peri-aortic and peri-iliac lymph nodes. Joshua et al (4) reported a case in which
$\mathrm{CT}$ demonstrated the presence of a rectal tumor originating from the anterior wall of the rectum, extending into the peri-rectal fat and seminal vesicles. Liver and lymph-node involvement has been observed in 70-80\% of patients in the early stages of extrapulmonary small cell carcinoma (16). A meta-analysis by Brenner et al (9) suggested that extrapulmonary small cell carcinoma arising from the gastrointestinal tract exhibits a common pattern of spread: First to the liver, followed by metastasis to the lymph nodes and bone marrow. In the present study, 3 patients presented with multiple liver metastases, 2 patients with multiple pulmonary metastases, 1 patient with multiple bone metastases and 1 patient with lymph nodes metastases in the perirectal fat spaces, inguinal region, hepatic hilar region, retroperitoneal, mediastinum and hilus pulmonis. Therefore, together, these results indicate that small cell carcinoma of the rectum is more likely to metastasize to the liver, pulmonary, lymph nodes and bone.

Due to the high incidence of distant metastases, surgery has been recommended as the primary palliative treatment for extrapulmonary small cell carcinoma; however, a multimodality approach with combined chemotherapy and radiation therapy has been advocated to improve patient survival time (5). Spiliopoulou et al (15) divided extrapulmonary small cell carcinoma of the rectum into two major groups, limited disease or extensive disease, based on whether the disease extent could be covered by an acceptable radiotherapy portal. The treatment options for extensive disease are systemic chemotherapy or supportive care. The treatment approach for limited disease is not clear; certain physicians suggest local treatment using surgery or radiotherapy, while others suggest multimodality approaches or chemotherapy alone (15). In an additional study of 81 patients with extrapulmonary small cell carcinoma, the majority of patients presented with limited disease, and the combination of chemotherapy and radiation therapy was observed to be as effective as surgery (17). It has also been suggested that local control of extrapulmonary small cell carcinoma of the rectum may be achieved with multidrug chemotherapy and radiation therapy, without the requirement for radical surgery (18). Surgery should only be performed on tumors of small dimensions, and a range of non-surgical treatments should be administered to patients with advanced-stage disease (19). In the present study, 1 patient (stage IVB), who underwent an exploratory laparotomy, sigmoidostomy and post-operative chemotherapy, succumbed to the carcinoma $\sim 2$ months after discharge from hospital. Another patient (stage IVB), who received no treatment, succumbed $\sim 1$ month after discharge from hospital, while the remaining 2 patients were lost to follow-up.

In conclusion, small cell carcinoma of the rectum is most likely to metastasize to the liver, pulmonary, lymph nodes and bone. Distinguishing features identified by CT and MRI include the thickening of the rectal wall, the presence of soft-tissue tumors, tumor local invasion, lymphadenopathy and distant metastasis. Pre-operative CT and MRI are required as an aid in selecting the correct treatment management and for the prognosis assessment of patients.

\section{Acknowledgements}

The authors would like to thank Professor Lizhi Liu (Medical Imaging and Minimally Invasive Interventional Center, Sun 
Yat-sen University Cancer Center; State Key Laboratory of Oncology in South China; Collaborative Innovation Center for Cancer Medicine, Guangzhou, China) for editing the original manuscript.

\section{References}

1. Lee SS, Ha HK, Kim AY, Kim TK, Kim PN, Yu E, Lee MG, Myung SJ, Jung HY, Kim JH and Min YI: Primary extrapulmonary small cell carcinoma involving the stomach or duodenum or both: Findings on CT and barium studies. AJR Am J Roentgenol 180: 1325-1329, 2003

2. Ihtiyar E, Algin C, Isiksoy S and Ates E: Small cell carcinoma of rectum: A case report. World J Gastroenterol 11: 3156-3158, 2005.

3. Clery AP, Dockerty MB and Waugh JM: Small-cell carcinoma of the colon and rectum. A clinicopathologic study. Arch Surg 83: 164-172, 1961.

4. Joshua AM, Adams D, McKenzie P, Solomon M and Clarke SJ: Small blue cell tumors of the rectum. Case 2. Small-cell carcinoma of the rectum. J Clin Oncol 23: 912-913, 2005.

5. Levine MS, Pantongrag-Brown L, Buck JL, Buetow PC, Lowry MA and Sobin LH: Small-cell carcinoma of the esophagus: Radiographic findings. Radiology 199: 703-705, 1996.

6. National Comprehensive Cancer Network (NCCN): NCCN Clinical Practise Guidelines in Oncology. Rectal Cancer. Version 2.2015. NCCN, Fort Washington, PA, p39, 2015.

7. Saclarides TJ, Szeluga D and Staren ED: Neuroendocrine cancers of the colon and rectum. Results of a ten-year experience. Dis Colon Rectum 37: 635-642, 1994.

8. Wong YN, Jack RH, Mak V, Henrik M and Davies EA: The epidemiology and survival of extrapulmonary small cell carcinoma in South East England, 1970-2004. BMC Cancer 9: 209, 2009.
9. Brenner B, Tang LH, Klimstra DS and Kelsen DP: Small-cell carcinomas of the gastrointestinal tract: A review. J Clin Oncol 22: 2730-2739, 2004.

10. Brenner B, Shah MA, Gonen M, Klimstra DS, Shia J and Kelsen DP: Small-cell carcinoma of the gastrointestinal tract: A retrospective study of 64 cases. Br J Cancer 90: 1720-1726, 2004.

11. Frazier SR, Kaplan PA and Loy TS: The pathology of extrapulmonary small cell carcinoma. Semin Oncol 34: 30-38, 2007.

12. Vilor M, Tsutsumi Y, Osamura RY, Tokunaga N, Soeda J, Ohta M, Nakazaki H, Shibayama Y and Ueno F: Small cell neuroendocrine carcinoma of the rectum. Pathol Int 45: 605-609, 1995.

13. Mills SE, Allen MS Jr and Cohen AR: Small-cell undifferentiated carcinoma of the colon. A clinicopathological study of five cases and their association with colonic adenomas. Am J Surg Pathol 7: 643-651, 1983.

14. Damjanov I, Amenta PS and Bosman FT: Undifferentiated carcinoma of the colon containing exocrine, neuroendocrine and squamous cells. Virchows Arch A Pathol Anat Histopathol 401: 57-66, 1983.

15. Spiliopoulou P, Panwar U and Davidson N: Rectal small cell carcinoma: A case report and review of the literature. Case Rep Oncol 4: 475-480, 2011.

16. Sterling RK: Ectopic ACTH syndrome associated with anorectal carcinoma. Report of a case and review of the literature. Dig Dis Sci 38: 955-959, 1993.

17. Galanis E, Frytak S and Lloyd RV: Extrapulmonary small cell carcinoma. Cancer 79: 1729-1736, 1997.

18. Robidoux A, Monté M, Heppell J and Schürch W: Small-cell carcinoma of the rectum. Dis Colon Rectum 28: 594-596, 1985.

19. Shirouzu K, Morodomi T, Isomoto H, Yamauchi Y, Kakegawa T and Morimatsu M: Small-cell carcinoma of the rectum. Clinicopathologic study. Dis Colon Rectum 28: 434-439, 1985. 